\title{
Temperature Effects in Acoustic Resonators
}

\author{
M. Červenka, M. Bednařík, P. Koníček \\ This paper deals with problems of nonlinear standing waves in axisymetrically shaped acoustic resonators where a mean temperature is \\ distributed along the axis.
}

Keywords: nonlinear standing wave, acoustic resonator, temperaturechangeover.

\section{Introduction}

The mean temperature distribution in resonators with high amplitudes of the acoustic field is caused by thermodynamic state changes due to the mean pressure and the density changeover, and in addition due to the conversion of acoustic energy into heat due to the viscous losses. The mean temperature distribution needs to be known in some appliances, such as thermoacoustic engines. The one-dimensional model equation for nonlinear standing waves of the $2^{\text {nd }}$ order including a viscous boundary layer is modified to describe the acoustic standing waves in a resonator with a longitudinal distribution of mean temperature. It is assumed that the mean temperature changes are small.

\section{Model equations}

Let us consider a one-dimensional acoustic field in an axisymetrically shaped gas-filled resonator driven by means of an external force. The model equation describing the acoustic field is derived from the basic equations of fluid mechanics. The one-dimensional form of these equations with terms up to the $2^{\text {nd }}$ order is presented here: Navier-Stokes equation, see [1],

$$
\begin{aligned}
\rho_{0} \frac{\partial v}{\partial t}+\rho^{\prime} \frac{\partial v}{\partial t}+\frac{\rho_{0}}{2} \frac{\partial v^{2}}{\partial x}= & -\rho_{0} a-\rho^{\prime} a-\frac{\partial p}{\partial x}+ \\
& +\left(\zeta+\frac{4}{3} \eta\right) \frac{\partial}{\partial x}\left[\frac{1}{r^{2}} \frac{\partial}{\partial x}\left(r^{2} v\right)\right],
\end{aligned}
$$

the continuity equation taking into account the boundary layer, see $[2,3]$,

$$
\begin{aligned}
\frac{\partial \rho^{\prime}}{\partial t}+\frac{\rho_{0}}{r^{2}} \frac{\partial}{\partial x}\left(r^{2} v\right) & +\frac{\rho^{\prime}}{r^{2}} \frac{\partial}{\partial x}\left(r^{2} v\right)+v \frac{\partial \rho^{\prime}}{\partial x}= \\
& =\frac{2}{r^{3}} \sqrt{v_{0}}\left(1+\frac{\gamma-1}{\sqrt{\operatorname{Pr}}}\right) \rho_{0} \frac{\partial^{\frac{1}{2}} r^{2} v}{\partial t^{-\frac{1}{2}} \partial x},
\end{aligned}
$$

where the fractional derivative represents an integrodifferential operator

$$
\frac{\partial^{-\frac{1}{2}} f}{\partial t^{-\frac{1}{2}}}=\frac{1}{\sqrt{\pi}} \int_{-\infty}^{t} f\left(t^{\prime}\right) \frac{\mathrm{d} t^{\prime}}{\sqrt{t-t^{\prime}}}
$$

and the thermodynamic state equation taking into account heat conductivity and mean temperature change, see [4],

$$
\begin{aligned}
p^{\prime} & =c^{2} \rho^{\prime}+c_{p} \rho_{0}(\gamma-1) \Delta \bar{\Theta}+ \\
& +\frac{c_{0}^{2}}{2 \rho_{0}}(\gamma-1) \rho^{\prime 2}-\kappa\left(\frac{1}{c_{V}}-\frac{1}{c_{p}}\right) \frac{1}{r^{2}} \frac{\partial}{\partial x}\left(r^{2} v\right),
\end{aligned}
$$

where $\rho^{\prime}, p^{\prime}, v$ are acoustic density, pressure and velocity, $p_{0}, \rho_{0}, \Theta_{0}$ are equilibrium state pressure, density and temperature, $\bar{\Theta}$ is mean temperature, $\Delta \bar{\Theta}=\bar{\Theta}-\Theta_{0}, a=a(t)$ is the driving acceleration, $x$ is the spatial coordinate along the resonant cavity, $t$ is time, $r=r(x)$ is radius of the resonator, $\gamma=c_{\mathrm{p}} / c_{V}$ is ratio of specific heats at constant pressure and volume, $\kappa$ is the coefficient of thermal conduction, $v_{0}$ is kinematic viscosity, $\zeta, \eta$ are the coefficients of bulk and shear viscosity, Pr is the Prandtl number, $c_{0}$ is the small-signal sound speed due to equilibrium temperature $\Theta_{0}$ and $c$ is the small-signal sound speed due to changed mean temperature defined as

$$
c^{2}=c_{0}^{2}\left(1+\frac{\Delta \bar{\Theta}}{\Theta_{0}}\right)=c_{0}^{2} \frac{\bar{\Theta}}{\Theta_{0}} .
$$

After linearization, from equations (1), (2) and (4) we obtain the following relations with terms of the $1^{\text {st }}$ order

$$
\begin{aligned}
p^{\prime} & =-\rho_{0}\left(\frac{\partial \varphi}{\partial t}+a x\right), \\
\rho^{\prime} & =-\frac{\rho_{0}}{c^{2}}\left(\frac{\partial \varphi}{\partial t}+a x\right)-\frac{c_{p}}{c^{2}} \rho_{0}(\gamma-1) \Delta \bar{\Theta}, \\
\frac{1}{r^{2}} \frac{\partial}{\partial x}\left(r^{2} \frac{\partial \varphi}{\partial x}\right) & =\frac{1}{c^{2}}\left(\frac{\partial^{2} \varphi}{\partial t^{2}}+x \frac{\mathrm{d} a}{\mathrm{~d} t}\right)
\end{aligned}
$$

where $\varphi$ is velocity potential, $v=\partial \varphi / \partial t$. For deriving the model equation, the following method is used: linearized equations, Eqs. (5), (6), (7), are substituted into terms of the $2^{\text {nd }}$ order and so the resulting error is of the $3^{\text {rd }}$ order. It is assumed that the mean temperature changeover is small and that its time and spatial derivatives are of the $2^{\text {nd }}$ order.

After eliminating acoustic density $\rho^{\prime}$ from Eq. (1) using Eq. (6), introducing velocity potential and after its integration with respect to the spatial coordinate $x$ we obtain the relation 


$$
\begin{aligned}
\rho_{0} \frac{\partial \varphi}{\partial t}- & \frac{\rho_{0}}{2 c^{2}}\left(\frac{\partial \varphi}{\partial t}+a x\right)^{2}-\frac{c_{p}}{c^{2}} \rho_{0}(\gamma-1) \Delta \bar{\Theta}\left(\frac{\partial \varphi}{\partial t}+a x\right)+ \\
+ & \frac{\rho_{0}}{2}\left(\frac{\partial \varphi}{\partial x}\right)^{2}=-\rho_{0} a x-p^{\prime}+\frac{\zeta+4 \eta / 3}{c^{2}}\left(\frac{\partial^{2} \varphi}{\partial t^{2}}+x \frac{\mathrm{d} a}{\mathrm{~d} t}\right)
\end{aligned}
$$

We can eliminate pressure $p^{\prime}$ from Eq. (8) using Eq. (4). Thus we get

$$
\begin{aligned}
& \rho_{0} \frac{\partial \varphi}{\partial t}-\frac{\rho_{0}}{2 c^{2}}\left(\frac{\partial \varphi}{\partial t}+a x\right)^{2}-\frac{c_{p}}{c^{2}} \rho_{0}(\gamma-1) \bar{\Theta}\left(\frac{\partial \varphi}{\partial t}+a x\right)+ \\
& +\frac{\rho_{0}}{2}\left(\frac{\partial \varphi}{\partial x}\right)^{2}=-c^{2} \rho^{\prime}-c_{p} \rho_{0}(\gamma-1) \Delta \bar{\Theta}-\rho_{0} a x+ \\
& +\frac{b}{c^{2}}\left(\frac{\partial^{2} \varphi}{\partial t^{2}}+x \frac{\mathrm{d} a}{\mathrm{~d} t}\right)-\frac{\rho_{0} c_{0}^{2}}{2 c^{4}}(\gamma-1)\left[\frac{\partial \varphi}{\partial t}+a x+c_{p}(\gamma-1) \Delta \bar{\Theta}\right]^{2} .
\end{aligned}
$$

After differentiating Eq. (9) with respect to time, we eliminate the time derivative $\partial \rho^{\prime} / \partial t$ using Eq. (2), thereby obtaining a model equation describing the nonlinear standing waves in an acoustic resonator with a spatial distribution of mean temperature. The derived model equation has the form

$$
\begin{gathered}
\frac{\partial^{2} \varphi}{\partial t^{2}}-c_{0}^{2} \frac{\bar{\Theta}}{\Theta_{0}} \frac{1}{r^{2}} \frac{\partial}{\partial x}\left(r^{2} \frac{\partial \varphi}{\partial x}\right)+\frac{\partial}{\partial t}\left(\frac{\partial \varphi}{\partial x}\right)^{2}=-x \frac{\mathrm{d} a}{\mathrm{~d} t}+ \\
-a \frac{\partial \varphi}{\partial x}+\frac{\gamma-1}{2 c_{0}^{2}}\left(\frac{\Theta_{0}}{\bar{\Theta}}\right)^{2} \frac{\partial}{\partial t}\left(\frac{\partial \varphi}{\partial t}+a x\right)^{2}+ \\
+\frac{b}{\rho_{0} c_{0}^{2}} \frac{\Theta_{0}}{\bar{\Theta}}\left(\frac{\partial^{3} \varphi}{\partial t^{3}}+x \frac{\mathrm{d}^{2} a}{\mathrm{~d} t^{2}}\right)+ \\
-\frac{2}{r} \sqrt{v_{0}}\left(1+\frac{\gamma-1}{\sqrt{\operatorname{Pr}}}\right)\left(\frac{\partial^{\frac{3}{2}} \varphi}{\partial t^{\frac{3}{2}}}+x \frac{\mathrm{d}^{\frac{1}{2}} a}{\mathrm{~d} t^{\frac{1}{2}}}\right)+ \\
-\frac{c_{p}}{c_{0}^{2}}(\gamma-1)^{2} \Delta \bar{\Theta}\left(\frac{\Theta_{0}}{\bar{\Theta}}\right)^{2}\left(\frac{\partial^{2} \varphi}{\partial t^{2}}+x \frac{\mathrm{d} a}{\mathrm{~d} t}\right)
\end{gathered}
$$

where $b=\zeta+4 \eta / 3+\kappa\left(1 / c_{V}-1 / c_{\mathrm{p}}\right)$ is the diffusity of sound.

This model equation is written in the coordinates moving together with the resonator body, consequently the boundary conditions have the form

$$
\frac{\partial \varphi}{\partial x}=v=0
$$

for $x=0$ and $x=\ell$, where $\ell$ is the resonator cavity length.

The acoustic pressure and density can be calculated from the numerical solution of Eq. (10), applicable equations are derived from relations (8) and (9)

$$
\begin{aligned}
p^{\prime} & =-\rho_{0} \frac{\partial \varphi}{\partial t}-\rho_{0} a x-\frac{\rho_{0}}{2}\left(\frac{\partial \varphi}{\partial x}\right)^{2}+\frac{\rho_{0}}{2 c_{0}^{2}} \frac{\Theta_{0}}{\bar{\Theta}}\left(\frac{\partial \varphi}{\partial t}+a x\right)^{2}+ \\
& +\frac{1}{c_{0}^{2}}\left(\zeta+\frac{4}{3} \eta\right) \frac{\Theta_{0}}{\bar{\Theta}}\left(\frac{\partial^{2} \varphi}{\partial t^{2}}+x \frac{\mathrm{d} a}{\mathrm{~d} t}\right)+ \\
& +\frac{c_{p}}{c_{0}^{2}} \rho_{0}(\gamma-1) \Delta \bar{\Theta} \frac{\Theta_{0}}{\bar{\Theta}}\left(\frac{\partial \varphi}{\partial t}+a x\right),
\end{aligned}
$$

$$
\begin{aligned}
& \rho^{\prime}=-\frac{\rho_{0}}{c_{0}^{2}} \frac{\Theta_{0}}{\bar{\Theta}} \frac{\partial \varphi}{\partial t}-\frac{\rho_{0}}{c_{0}^{2}} \frac{\Theta_{0}}{\bar{\Theta}} a x-\frac{\rho_{0}}{2 c_{0}^{2}} \frac{\Theta_{0}}{\bar{\Theta}}\left(\frac{\partial \varphi}{\partial x}\right)^{2}+ \\
& -\frac{c_{p}}{c_{0}^{2}} \rho_{0}(\gamma-1) \Delta \bar{\Theta} \frac{\Theta_{0}}{\bar{\Theta}}+\frac{c_{p}^{2}}{2 c_{0}^{4}} \rho_{0}(\gamma-1)^{3}(\Delta \bar{\Theta})^{2}\left(\frac{\Theta_{0}}{\bar{\Theta}}\right)^{3}+ \\
& +\frac{b}{c_{0}^{4}}\left(\frac{\Theta_{0}}{\bar{\Theta}}\right)^{2}\left(\frac{\partial^{2} \varphi}{\partial t^{2}}+x \frac{\mathrm{d} a}{\mathrm{~d} t}\right)+\frac{\rho_{0}}{c_{0}^{4}}\left[1-(\gamma-1) \frac{\Theta_{0}}{\bar{\Theta}}\right]\left(\frac{\Theta_{0}}{\bar{\Theta}}\right)^{2} \times \\
& \times\left[\frac{1}{2}\left(\frac{\partial \varphi}{\partial t}+a x\right)^{2}+c_{p}(\gamma-1) \Delta \bar{\Theta}\left(\frac{\partial \varphi}{\partial t}+a x\right)\right] .
\end{aligned}
$$

A one-dimensional model equation describing the temperature distribution is derived from the energy equation for an ideal gas, see [5],

$$
\begin{aligned}
\rho c_{V} \frac{\mathrm{d} \Theta}{\mathrm{d} t} & =-p \frac{\partial v_{i}}{\partial x_{i}}+\eta\left(\frac{\partial v_{i}}{\partial x_{j}}+\frac{\partial v_{j}}{\partial x_{i}}-\frac{2}{3} \delta_{i j} \frac{\partial v_{\ell}}{\partial x_{\ell}}\right) \frac{\partial v_{i}}{\partial x_{j}}+ \\
& +\zeta \frac{\partial v_{\ell}}{\partial x_{\ell}} \delta_{i j} \frac{\partial v_{i}}{\partial x_{j}}+\frac{\partial}{\partial x_{j}}\left(\kappa \frac{\partial \Theta}{\partial x_{j}}\right),
\end{aligned}
$$

where $\delta_{i j}$ is the Kronecker delta, indices $i, j, \ell$ go from 1 to 3 and $v_{i}$ represents three components of the acoustic velocity vector. A one-dimensional form of Eq. (13) is obtained using the relation for one-dimensional divergence in an axisymetrically shaped waveguide, see [1],

$$
\begin{aligned}
\rho c_{V} \frac{\mathrm{d} \Theta}{\mathrm{d} t} & =-\frac{p}{r^{2}} \frac{\partial}{\partial x}\left(r^{2} v\right)+\left(\zeta+\frac{4}{3} \eta\right)\left[\frac{1}{r^{2}} \frac{\partial}{\partial x}\left(r^{2} v\right)\right]^{2}+ \\
& +\frac{\kappa}{r^{2}} \frac{\partial}{\partial x}\left(r^{2} \frac{\partial \Theta}{\partial x}\right)+\kappa \frac{\partial^{2} \Theta}{\partial y^{2}},
\end{aligned}
$$

where $y$ is the spatial coordinate normal to the resonator wall. The last term of Eq. (14) describes the heat flow through the boundary layer to the resonator wall.

For the temperature in the resonator cavity, we can write $\Theta(x, y, t)=\Theta_{B}(x, y, t)+\Theta_{M}(x, t)$, where $\Theta_{B}$ is the temperature in the boundary layer and $\Theta_{M}$ is the mainstream temperature. It is easy to show, see [3], that

$$
\Theta_{B}=\sum_{k=-\infty}^{\infty} \Theta_{E k} \exp \left[-\sqrt{\frac{j k \omega \operatorname{Pr}}{v_{0}} y}\right] e^{\mathrm{j} k \omega t}
$$

and thus

$\left.\frac{\partial \Theta_{B}}{\partial y}\right|_{y=0}=-\sqrt{\frac{\operatorname{Pr}}{v_{0}}} \sum_{k=-\infty}^{\infty} \sqrt{\mathrm{j} k \omega} \Theta_{E k} e^{\mathrm{j} k \omega t}=-\sqrt{\frac{\operatorname{Pr}}{v_{0}}} \frac{\mathrm{d}^{\frac{1}{2}} \Theta}{\mathrm{d} t^{\frac{1}{2}}}$.

Integrating Eq. (14) with respect to the resonator cross-section with help of the divergence theorem yields

$$
\begin{gathered}
\iint_{\mathcal{S}}\left\{\rho c_{V} \frac{\mathrm{d} \Theta}{\mathrm{d} t}+\frac{p}{r^{2}} \frac{\partial}{\partial x}\left(r^{2} v\right)-\left(\zeta+\frac{4}{3} \eta\right)\left[\frac{1}{r^{2}} \frac{\partial}{\partial x}\left(r^{2} v\right)\right]^{2}+\right. \\
\left.-\frac{\kappa}{r^{2}} \frac{\partial}{\partial x}\left(r^{2} \frac{\partial \Theta}{\partial x}\right)\right\} \mathrm{d} \mathcal{S}=\kappa \oint_{\mathcal{C}} \frac{\partial \Theta}{\partial y} \mathrm{~d} \ell,
\end{gathered}
$$

where $\mathcal{S}$ is the resonator cross-section and $\mathcal{C}$ is its boundary.

After substitution of relation (16) into Eq. (17) and calculation of the integrals (acoustic quantities are assumed to be constant with respect to the integration domain), we obtain the model equation for the temperature distribution 


$$
\begin{aligned}
\left(\rho^{\prime}+\rho_{0}\right) c_{V} \frac{\mathrm{d} \Theta}{\mathrm{d} t} & =-\left(p^{\prime}+p_{0}\right) \frac{1}{r^{2}} \frac{\partial}{\partial x}\left(r^{2} \frac{\partial \varphi}{\partial x}\right)+ \\
& +\left(\zeta+\frac{4}{3} \eta\right)\left[\frac{1}{r^{2}} \frac{\partial}{\partial x}\left(r^{2} \frac{\partial \varphi}{\partial x}\right)\right]^{2}+ \\
& +\frac{\kappa}{r^{2}} \frac{\partial}{\partial x}\left(r^{2} \frac{\partial \Theta}{\partial x}\right)-\frac{2 \kappa}{r} \sqrt{\frac{\operatorname{Pr}}{v_{0}}} \frac{\partial^{\frac{1}{2}} \Theta}{\partial t^{\frac{1}{2}}} .
\end{aligned}
$$

Eq. (10) is solved numerically in the frequency domain, and driving acceleration $a$ is assumed to be periodic. Owing to the numerical instability of Eq. (18) solved in the frequency domain, the mean temperature change is estimated from the thermodynamic state equation (it is assumed that all the heat generated in the resonator cavity due to viscous losses is conducted out through the resonator cavity walls) as

$$
\bar{\Theta}=\Theta_{0}\left(1+\frac{\overline{p^{\prime}}}{p_{0}}-\frac{\vec{\rho}}{\rho_{0}}\right) .
$$

\section{Numerical results and conclusions}

Fig. 1 shows the spatial distribution of the mean temperature in the cylindrical, conical and bulb resonator due to the medium thermodynamic state change in an intensive sound field. The temperature was estimated using Eq. (19). The resonator driving acceleration $a=3000 \mathrm{~m} \cdot \mathrm{s}^{-2}$, the driving frequency agrees with the $1^{\text {st }}$ natural frequency of the resonant cavities, the length of the resonators $\ell=0.17 \mathrm{~m}$, the radiuses of the resonators are:

- cylindrical resonator $-r(x)=0.01 \mathrm{~m}$,
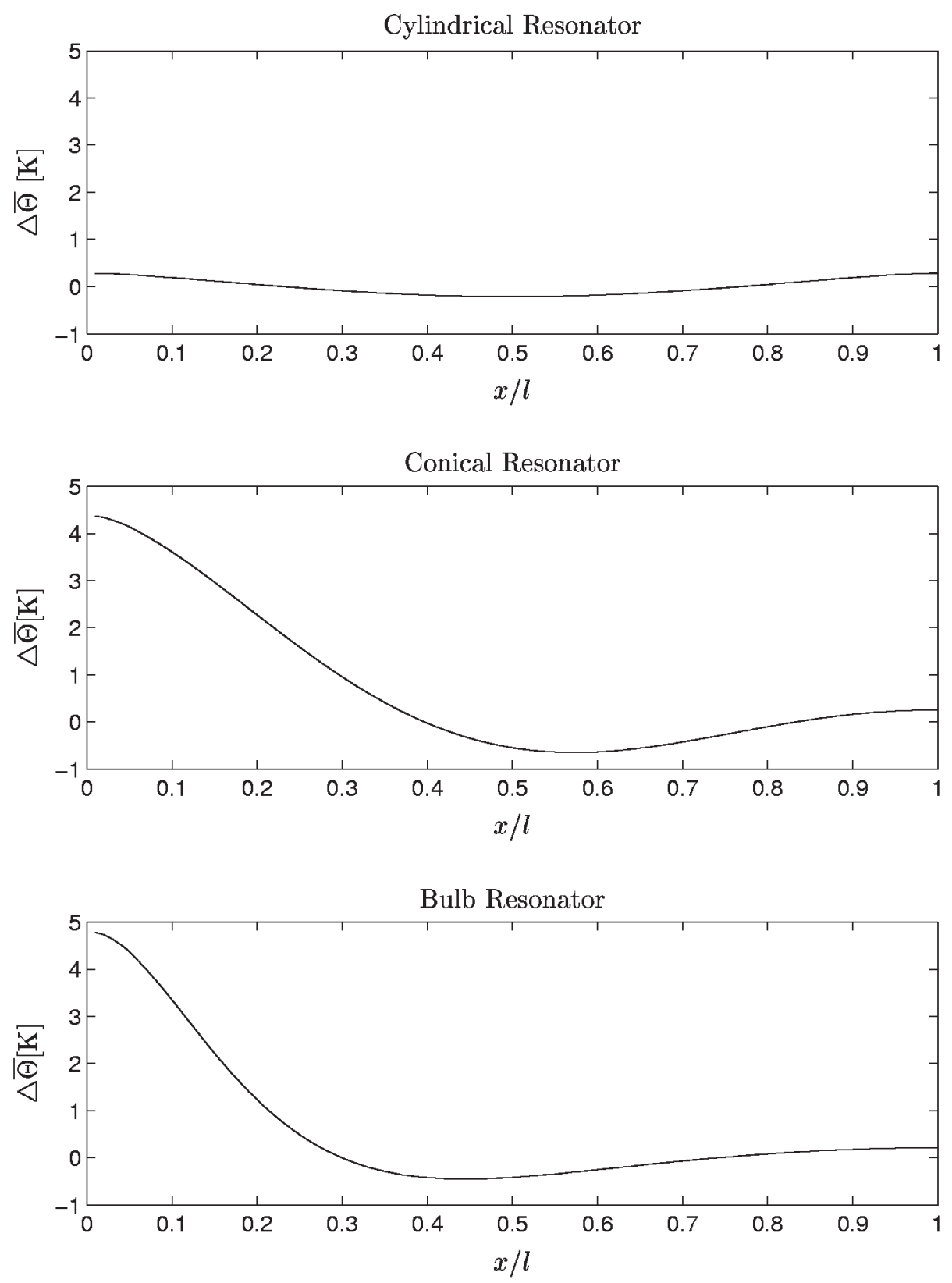

Fig. 1: Mean temperature distribution in a cylindrical, conical and bulb resonator 


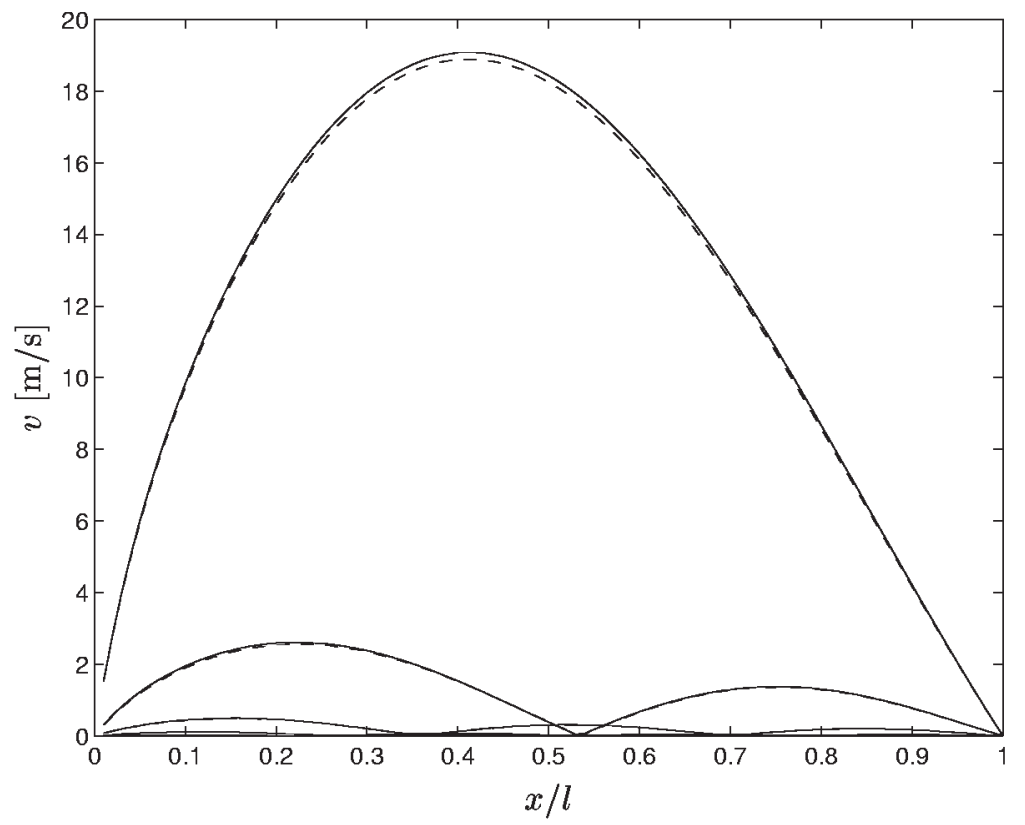

Fig. 2: Distribution of the acoustic velocity spectrum in conical resonator. Dashed line: temperature distribution is taken into account, solid line: temperature distribution is not taken into account.

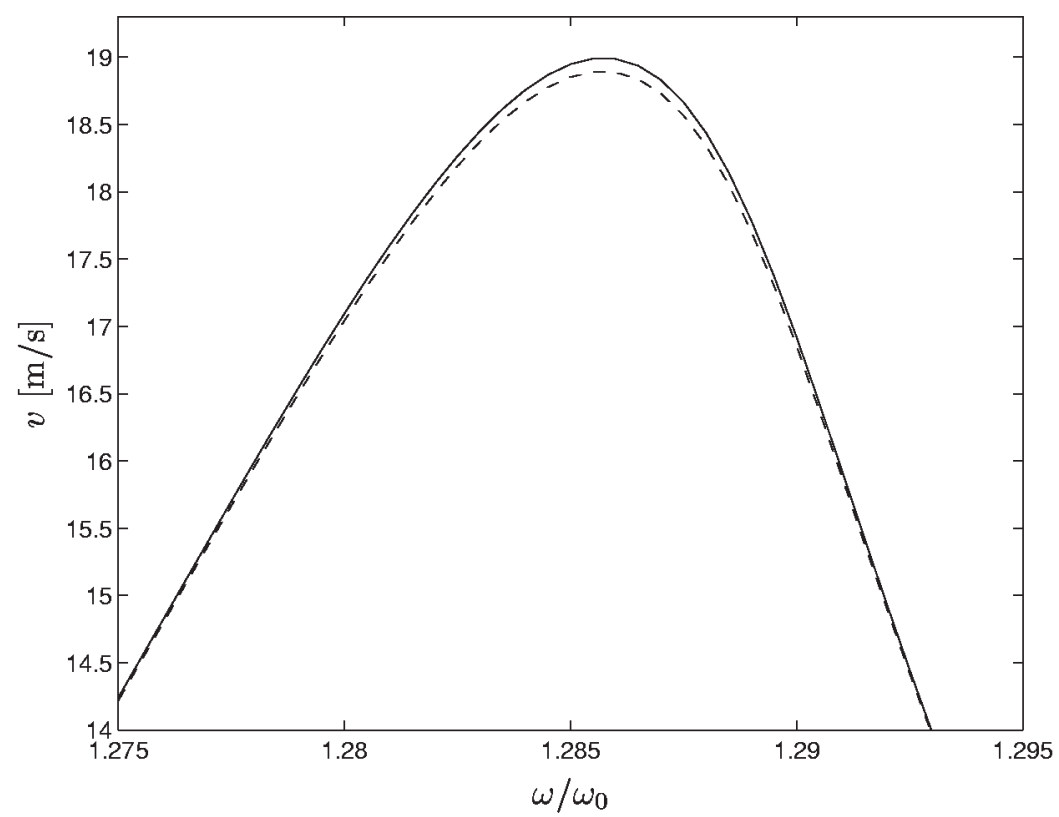

Fig. 3: Frequency characteristics of a conical resonator. Dashed line: temperature distribution is taken into account, solid line: temperature distribution is not taken into account.

- conical resonator $-r(x)=0.0056+0.268 x \mathrm{~m}$,

- bulb resonator $-r(x)=0.003 \sin (\pi x / \ell) \exp (3 x / \ell)+0.005 \mathrm{~m}$.

It can be seen that the mean temperature is found near the pressure antinodes.

Fig. 2 compares the acoustic velocity spectrum distribution in a conical resonator where the influence of the temperature distribution is taken into account (dashed line) and where it is not taken into account (solid line). Fig. 3 compares the frequency characteristics for the same resonator. The numerical results show a slight resonant frequency shift and a waveform changeover if these mean temperature changes in the medium are taken into account.

Fig. 4 shows an example of a thermoacoustic engine. The upper figure shows the mean temperature distribution, the bottom figure compares the acoustic velocity spectrum if the mean temperature distribution is taken into account (dashed 

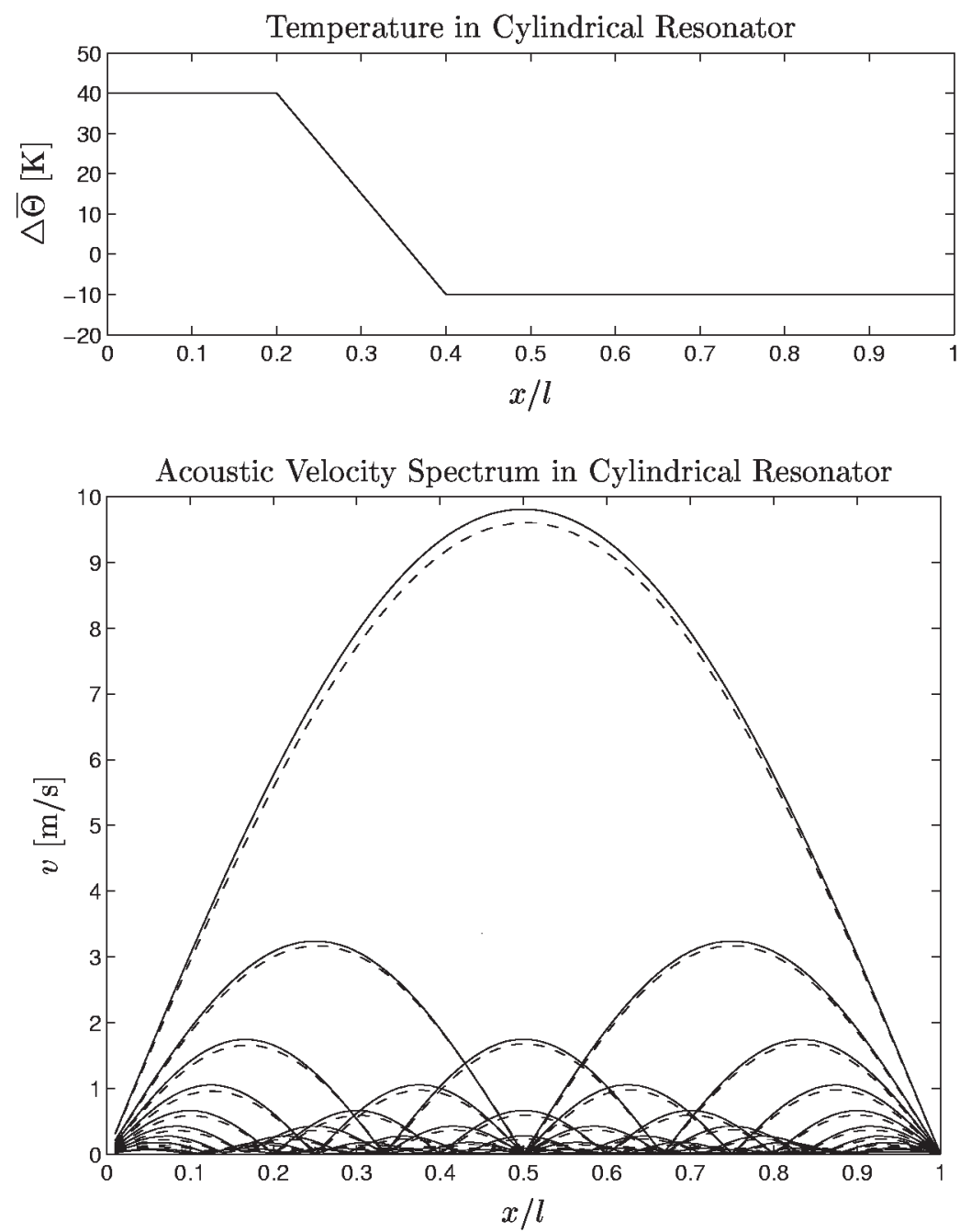

Fig. 4: Mean temperature distribution in cylindrical resonator due to external heating and cooling (upper figure), comparison of acoustic velocity spectra in the resonator if the mean temperature distribution is taken into account (dashed line) and if the mean temperature distribution is not taken into account (solid line).

line) and if the mean temperature distribution is not taken into account (solid line).

\section{References}

[1] Ilinskii, Y. A., Lipkens, B., Lucas, T. S., Van Doren, T. W., Zabolotskaya, E. A.: Nonlinear standing waves in an acoustical resonator. J. Acoust. Soc. Am. Vol. 104, 1998, p. 2664-2674.

[2] Bednařík, M., Červenka, M.: Nonlinear Waves in Resonators. Proc. of the $15^{\text {th }}$ ISNA, Goettingen (Germany), 1999.

[3] Chester, W.: Resonant oscillations in closed tubes. J. Fluid Mech., Vol. 18, 1964, p. 44-64.

[4] Makarov, S., Ochmann, M.: Nonlinear and Thermoviscous Phenomena in Acoustics. Part I, ACUSTICA - Acta Acustica, Vol. 82, 1996, p. 579-605.

[5] Blackstock, D. T.: Fundamentals of physical acoustics. New York: John Wiley \& Sons, Inc., 2000, p. 77-84.
Ing. Milan Červenka phone: +420224353975 e-mail: cervenm3@feld.cvut.cz

Dr. Ing. Michal Bednařík phone: +420224352308 e-mail: bednarik@feld.cvut.cz

Dr. Mgr. Petr Koníček

phone: +420224352329

e-mail:konicek@feld.cvut.cz

Department of Physics

Czech Technical University in Prague

Faculty of Electrical Engineering

Technická 2

16627 Prague, Czech Republic 